\title{
Plasma control by modification of helicon wave propagation in low magnetic fields
}

\author{
T. Lafleur, ${ }^{\text {a) }}$ C. Charles, and R. W. Boswell \\ Space Plasma, Power and Propulsion Group, Research School of Physics and Engineering, \\ The Australian National University, Canberra, Australian Capital Territory 0200, Australia
}

(Received 6 May 2010; accepted 14 June 2010; published online 23 July 2010)

\begin{abstract}
By making use of nonuniform magnetic fields, it is shown experimentally that control of helicon wave propagation can be achieved in a low pressure $(0.08 \mathrm{~Pa})$ expanding plasma. The $m=1$ helicon waves are formed during a direct capacitive to wave mode transition that occurs in a low diverging magnetic field $\left(B_{0}<3 \mathrm{mT}\right)$. In this initial configuration, waves are prevented from reaching the downstream region, but slight modifications to the magnetic field allows the axial distance over which waves can propagate to be controlled. By changing the effective propagation distance in this way, significant modification of the density and plasma potential profiles can be achieved, showing that the rf power deposition can be spatially controlled as well. Critical to the modification of the wave propagation behavior is the magnetic field strength (and geometry) near the exit of the plasma source region, which gives electron cyclotron frequencies close to the wave frequency of 13.56 MHz. () 2010 American Institute of Physics. [doi:10.1063/1.3460351]
\end{abstract}

\section{INTRODUCTION}

Helicon wave discharges are often associated with very high ionization rates, and together with the high power coupling efficiencies attainable between the antenna and the plasma, are able to produce large densities $\left(10^{17}-10^{19} \mathrm{~m}^{-3}\right.$ for powers between a few 100 and several $1000 \mathrm{~W}$ ) over a range of gas pressures (from less than 0.1 to a few pascals). ${ }^{1-3}$ Critical for helicon mode initiation and subsequent wave propagation is the presence of a magnetic field $B_{0}$, which typically has strengths of tens to hundreds of millitesla. ${ }^{2,3}$ Theory predicts that the wavelength of a helicon wave is a function of the ratio $B_{0} / n$, and as the magnetic field is increased for a given wavelength, the plasma density $n$ increases approximately linearly. ${ }^{2,4}$ At low magnetic fields $(<10 \mathrm{mT})$, however, this proportionality no longer holds, and density peaks occurring over a narrow range of field values have been reported by several researchers. ${ }^{5-11}$ These low field peaks have been studied over a fairly wide range of power and pressure values, with the large majority of work done making use of uniform magnetic field geometries. The magnetic field present at the maximum of these density peaks has been shown experimentally ${ }^{9}$ and numerically ${ }^{12}$ to be approximately proportional to the wave frequency, and for frequencies of $13.56 \mathrm{MHz}$, occur at around $2-3 \mathrm{mT} .^{6,8,11}$ Associated with the density peaks of these low field helicons are corresponding peaks in the antenna loading resistance, ${ }^{4,9,12}$ showing that the power transfer efficiency between the antenna and the plasma increases. Chen ${ }^{4}$ has suggested that the formation of these peaks for $m=0$ antennas is caused by constructive interference between forward waves launched by the antenna and reflected waves from an end plate. This has been verified by Cho, ${ }^{12}$ who also showed that these peaks can be formed for $m=1$ antennas regardless of

\footnotetext{
${ }^{a)}$ Electronic mail: trevor.lafleur@anu.edu.au.
}

wave reflections; although wave reflections were seen to enhance the observed peaks. A number of other researchers have noted a correlation between the wave phase velocities and certain significant electron velocities ${ }^{5,6,10}$ (such as the velocity of electrons giving a peak in the ionization rate), which could lead to wave-particle interactions. ${ }^{10}$ Low field helicons are particularly attractive for plasma processing ${ }^{13,14}$ or space propulsion applications, ${ }^{15}$ where the small field needed reduces the required equipment and system hardware, while also having significantly lower power requirements (since the power consumption in any solenoids is proportional to $B_{0}^{2}$ ) or none at all if permanent magnets are used. While some work has been done on these low field helicons in nonuniform magnetic fields, ${ }^{7,15}$ the majority of work has typically focused on uniform fields. The use of nonuniform fields in helicon systems is of interest as it can lead to enhanced ionization rates and can substantially change the plasma and wave propagation properties when compared with uniform fields. ${ }^{16-18}$ In this paper, we report on helicon experiments performed in low nonuniform (diverging) magnetic fields $\left(B_{0}<2.5 \mathrm{mT}\right)$ and show that the presence of an electron cyclotron resonance (ECR) region in such fields can be used to significantly alter both the helicon wave propagation and plasma properties in the upstream and downstream regions of the reactor.

\section{APPARATUS}

Experiments are performed in the Piglet helicon reactor, shown schematically in Fig. 1. It consists of a $20 \mathrm{~cm}$ long, $13.6 \mathrm{~cm}$ diameter cylindrical Pyrex source tube, connected to a larger $32 \mathrm{~cm}$ diameter, $28.8 \mathrm{~cm}$ long aluminum diffusion chamber. The source tube is terminated at one end by a grounded metal grid to which a rotary/turbomolecular vacuum pump system is connected. Surrounding the source tube is a $10 \mathrm{~cm}$ long double-saddle field antenna, constructed from rectangular copper strips, $1.7 \mathrm{~mm}$ thick and $12 \mathrm{~mm}$ 


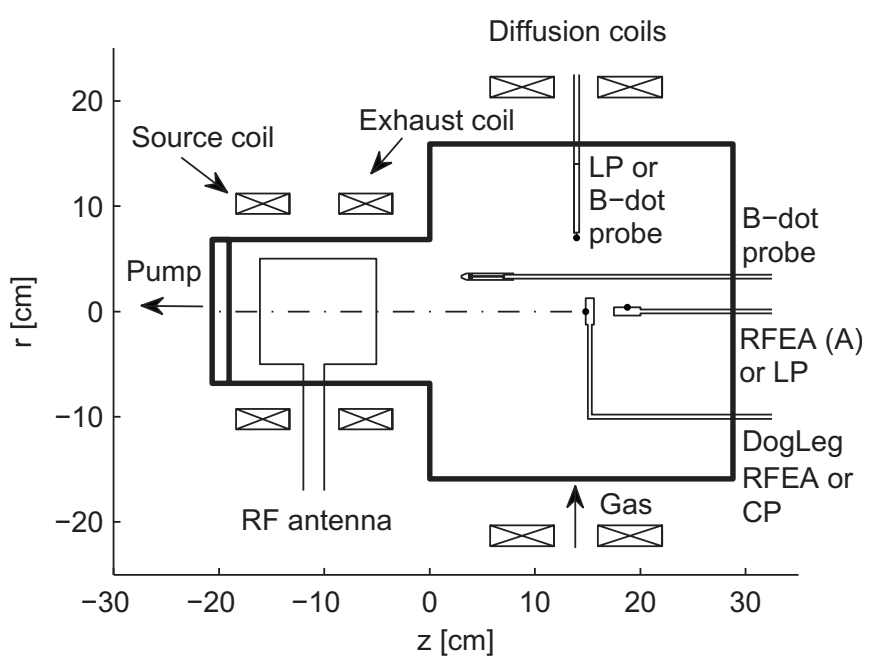

FIG. 1. The Piglet helicon reactor, showing the source region $(z<0 \mathrm{~cm})$, diffusion chamber $(z>0 \mathrm{~cm})$, magnetic field coils, and a representation of the insertion location of the diagnostic probes used.

wide, silver soldered together. It extends from $z=-16 \mathrm{~cm}$ to $z=-5 \mathrm{~cm}$ (where $z=0 \mathrm{~cm}$ is defined at the interface between the source tube and the diffusion chamber). The antenna is connected to a $\pi$ matching network and a rf power generator with an operating frequency of $13.56 \mathrm{MHz}$. The forward and reverse power to the matchbox is monitored using a directional coupler connected to an analog oscilloscope and is placed between the matching network and the rf generator. The Piglet reactor makes use of two pairs of magnetic field coils. The first pair surrounds the antenna/source tube, with each coil consisting of approximately 500 turns. The coil closest to the pump is termed the source coil, while the other coil is termed the exhaust coil. The second pair of coils is placed around the diffusion chamber, with each coil consisting of 280 turns. Argon gas is fed into the reactor through an inlet port in the side of the diffusion chamber, and the flow rate in SCCM (SCCM denotes standard cubic centimeters per minute) is set via a flow controller. The gas pressure is measured using a baratron pressure gauge attached to the backplate of the diffusion chamber, while an ion gauge measures the system base pressure (which is less than $0.3 \mathrm{mPa}$ ). A number of ports are present for diagnostic probes, both in the side, and within the backplate of the diffusion chamber (see Fig. 1).

\section{DIAGNOSTICS}

The plasma density is measured using a Langmuir probe (LP) consisting of a small $2 \mathrm{~mm}$ diameter nickel disk oriented perpendicular to "Piglet's" central axis and attached to the end of a hollow ceramic tube. The probe is operated in ion saturation mode with an applied bias voltage of $-45 \mathrm{~V}$, and the current is determined from the voltage drop across a $1 \mathrm{k} \Omega$ sense resistor. To establish the electron temperature, use is made of a rf compensated Langmuir probe (CP) previously described. ${ }^{19}$ The electron temperature is then established by fitting a straight line to the electron energy probability function, which is proportional to the second derivative of the collected $I V$ characteristic. ${ }^{20}$ The plasma

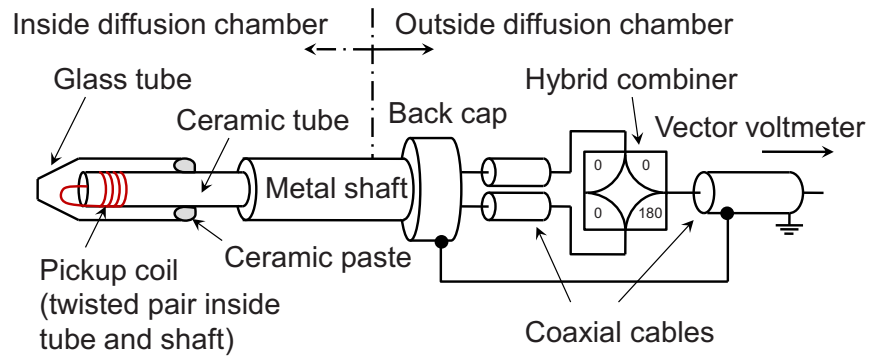

FIG. 2. (Color online) Schematic of a B-dot probe and associated circuitry used to measure the axial component $\left(B_{z}\right)$ of the wave fields. A vacuum feedthrough (not shown) is located at the position of the vertical dotted line. Parts of the probe to the left of the dotted line are situated inside the diffusion chamber, while parts to the right are situated outside. The metal shaft can translate axially through the feedthrough while still maintaining vacuum integrity.

potential in "Piglet" is measured using a retarding field energy analyzer (RFEA) [with orifice facing the walls of the reactor; RFEA (A) in Fig. 1], which has been previously described. $^{21-23}$ A second "dogleg" RFEA with its orifice facing the source tube exit is used to detect the presence of an ion beam in the downstream plasma. ${ }^{24}$ Measurements of the wave fields are made using a number of B-dot probes (a schematic of which is shown in Fig. 2), each consisting of a $4 \mathrm{~mm}$ diameter copper coil (with approximately 6 turns) supported by a small hollow ceramic tube. The output of the probe is connected to a hybrid combiner (which allows any electrostatic pickup to be separated from the magnetic signal $^{25}$ ) and an HP 8405A vector voltmeter, allowing the magnitude and phase to be determined using a reference voltage from the directional coupler connected to the matchbox. The B-dot probe design was checked for electrostatic pickup noise by making use of a Faraday cup ${ }^{26}$ and found to have a rejection ratio of around 100:1 at $13.56 \mathrm{MHz}$. Tests within the plasma showed that by rotating the B-dot probe by $180^{\circ}$, the signal magnitude obtained remained virtually the same, with only the sign changing. This gives us confidence that the probes are only measuring the inductive component of any fields present. By separating the electrostatic pickup from the magnetic signal, the hybrid combiner has the advantage of allowing an estimate of the error in the wave field measurements to be made. Using the known rejection ratio and by monitoring the electrostatic pickup, average uncertainties of around $2 \%-7 \%$ were obtained, except for certain measurements in the diffusion chamber where the magnetic signal was close to zero, and hence larger average uncertainties of around $20 \%-25 \%$ were observed.

\section{RESULTS}

\section{A. Low magnetic field helicon waves}

It has recently been shown that a density peak can be produced in the present reactor in a low nonuniform (diverging) magnetic field. ${ }^{27}$ In that work a pressure of $0.04 \mathrm{~Pa}$ and a rf power of $250 \mathrm{~W}$ were used, whereas the present work is conducted at a higher pressure of $0.08 \mathrm{~Pa}$ (the reasons for this are discussed later in Sec. IV B) and the same input power. With only the source coil operating (see Fig. 1), a 

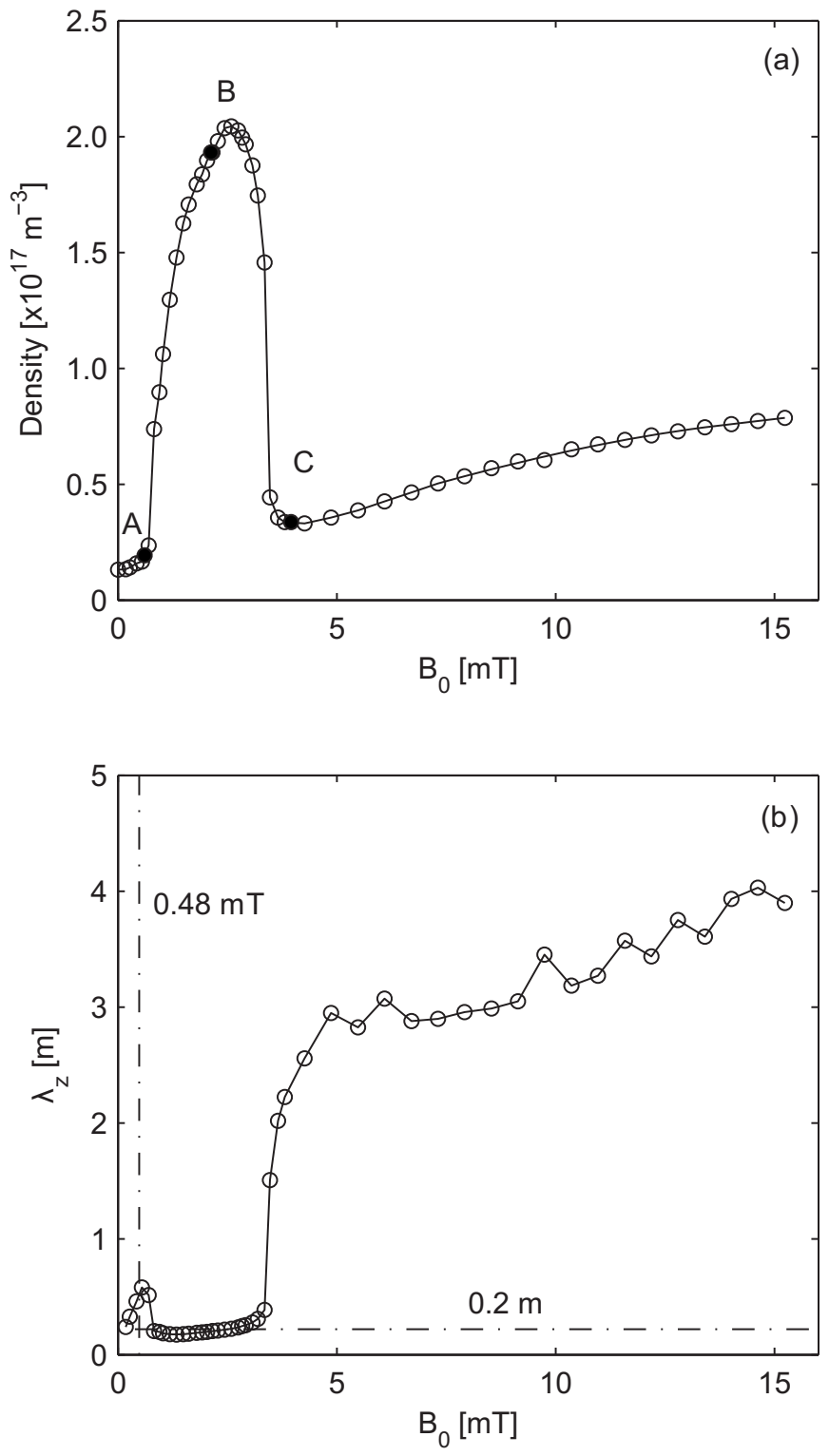

FIG. 3. (a) Plasma density within the source region $(z=-10 \mathrm{~cm})$ as a function of the maximum applied magnetic field (with only the source coil operating). Marked cases $\mathrm{A}$ and $\mathrm{C}$ show plasma conditions before and after the density peak, while case B corresponds to plasma conditions close to the maximum of the peak. (b) Hypothetical axial helicon wavelength $\lambda_{z}$ calculated from Eqs. (1) and (2) using densities and magnetic field values from (a). The vertical dotted line marks the magnetic field giving an electron cyclotron frequency of $13.56 \mathrm{MHz}$, while the horizontal dotted line at $0.2 \mathrm{~m}$ marks twice the antenna axial length.

large increase in plasma density in the source region is observed over a narrow range of magnetic field values $\left(0.6 \mathrm{mT}<B_{0}<3.5 \mathrm{mT}\right)$. Figure 3(a) shows the plasma density (obtained from the LP using an electron temperature of $6 \mathrm{eV}$ from the $\mathrm{CP}$ ) in the source region at position $z=-10 \mathrm{~cm}$ as a function of the maximum magnetic field present. Here it is seen that the peak density (close to point B in Fig. 3) at $B_{0}=3 \mathrm{mT}$ is more than an order of magnitude larger than that occurring before or after the density increase [points A and C in Fig. 3(a)]. It has been shown previously that in these low pressure plasmas a capacitive to inductive to wave mode transition is typically seen as the rf power or magnetic field is increased. ${ }^{28}$ However, at low magnetic fields, a direct capacitive to wave mode transition is sometimes observed. ${ }^{29}$

The dispersion relation for helicon waves in a bounded system is given by ${ }^{1}$

$$
\frac{k k_{z}}{k_{0}^{2}}=\frac{\omega_{p e}^{2}}{\omega \omega_{c e}},
$$

where $k=\sqrt{k_{z}^{2}+k_{\perp}^{2}}$ is the wave vector magnitude, $k_{z}$ and $k_{\perp}$ are the axial and radial wave vector components, $k_{0}=\omega / c, \omega$ is the wave angular frequency, $c$ is the speed of light in vacuum, $\omega_{p e}$ is the electron plasma frequency, and $\omega_{c e}$ is the electron cyclotron frequency. For a cylinder of radius $R$, Eq. (1) can be solved together with appropriate boundary conditions at the cylinder wall to establish the wave vector components $k_{z}$ and $k_{\perp}$. For an insulating wall, a commonly used boundary condition is that the radial current density amplitude is zero, leading to the condition that the radial component of the wave field $B_{r}$ must also vanish, ${ }^{30}$ which provides a relationship between $k_{z}$ and $k_{\perp}$ given by

$$
m k J_{m}\left(k_{\perp} R\right)+k_{z} R J_{m}^{\prime}\left(k_{\perp} R\right)=0
$$

where $m$ is the helicon wave azimuthal mode number, $J_{m}$ is a Bessel function of the first kind, and the prime denotes the derivative with respect to the radial coordinate. A more exact approach would solve for the wave fields inside the plasma filled cylinder, as well as the vacuum outside, and match the fields at the interface. ${ }^{31}$ As a first approximation, however, we ignore the vacuum fields (together with the axial inhomogenieties in the plasma density and magnetic field) and make use of Eq. (2), together with Eq. (1), to establish the wave vector components. It should be noted that the source and exhaust coil support structure is metallic and surrounds the Pyrex source tube, with only a small gap present, thus it is felt that the insulating boundary condition is a reasonable approximation. With the wave vector components established, the axial wavelength can be found from $\lambda_{z}=2 \pi / k_{z}$. Figure 3(b) shows a hypothetical helicon wavelength $\lambda_{z}$ obtained using the density and magnetic field values from Fig. 3(a) and assuming an azimuthal mode number $m=1$. Here we observe that the wavelength remains approximately constant at $0.2 \mathrm{~m}$ for all points within the density peak, while for points outside the peak, the wavelength is significantly higher. It is interesting to note that $0.2 \mathrm{~m}$ also corresponds to twice the average axial antenna length, which for a doublesaddle field antenna is a well known matching condition leading to efficient coupling between the antenna and helicon mode. ${ }^{1}$ At low pressures $(<0.1 \mathrm{~Pa})$, or low powers (less than a few hundred watts), the plasma density is often fairly low, and the applied magnetic field is usually too large for this matching condition to be attained. However, if a low magnetic field is present, then this wavelength matching condition can more easily be satisfied. The above discussion suggests that during the transition, the antenna matching efficiency has increased, a fact that is consistent with previous measurements of the effective antenna resistance, ${ }^{27}$ which shows a peak in the resistance well correlated with the observed density peak. This demonstrates that during the 

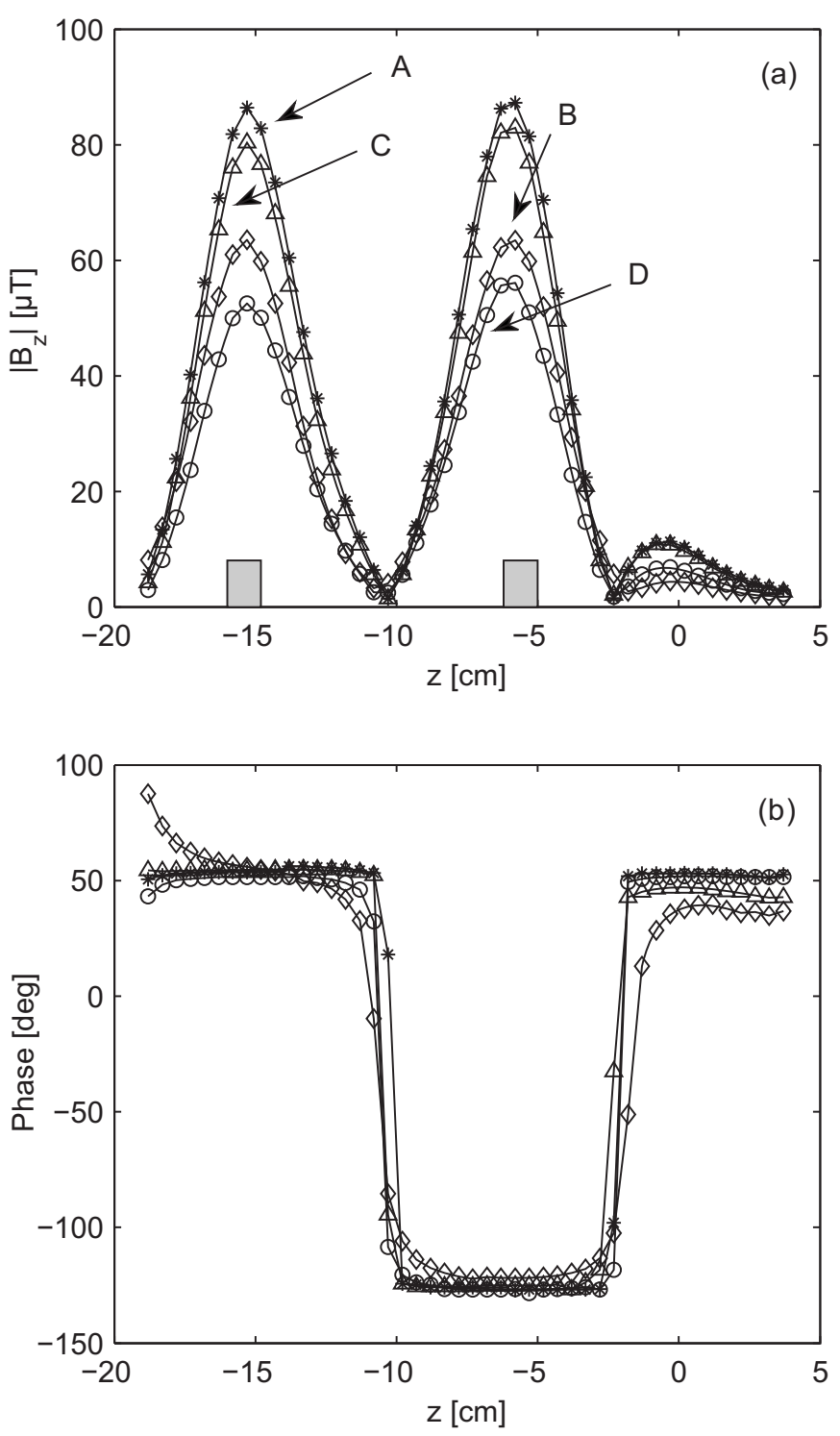

FIG. 4. (a) Axial profile of the amplitude of $B_{z}$ in the source region at $r=4.8 \mathrm{~cm}$ for case A (stars), case B (open diamonds), and case C (open triangles) in Fig. 3, together with the fields present with no plasma in the reactor (case D; open circles). The rectangles at $z=-15.5 \mathrm{~cm}$ and $z=-5.5 \mathrm{~cm}$ mark the location of the azimuthal straps of the double-saddle field antenna. (b) Phase variation of $B_{z}$ for the cases in (a).

mode transition, the power coupling efficiency increases, and a larger percentage of the input power is deposited within the plasma.

\section{B. Wave field measurements}

The results of Sec. IV A suggest that the observed density peak is a wave mode occurring over a narrow range of magnetic field values; however, in order to verify this, the wave fields need to be investigated. To do this, B-dot probe measurements are carried out for points marked A-C in Fig. 3(a). In order to estimate the magnitude of fields observed, the B-dot probe is calibrated with a small current loop. Figure 4 shows the magnitude and phase of the axial magnetic field component $B_{z}$ taken at a radial distance of $r=4.8 \mathrm{~cm}$ (the source tube radius is $6.8 \mathrm{~cm}$ ). As is seen, the general behavior for each of points A-C is essentially the same, with equal magnitude peaks occurring at $z=-4.5 \mathrm{~cm}$ and $z=-15.5 \mathrm{~cm}$, respectively, while field minima occur at $z=-10.5 \mathrm{~cm}$ and $z=-2.5 \mathrm{~cm}$. A smaller peak is also observed at $z=-0.5 \mathrm{~cm}$. From Fig. 4(b), it is seen that the phase remains essentially constant under each peak in Fig. 4(a), with a $\pi$ phase change occurring at each of the minima, highly suggestive of standing wave behavior. However, also shown in Fig. 4 are the fields (case D) present (at an input power of $100 \mathrm{~W}$ ) with no gas in the reactor (and hence no plasma). Once again, the same behavior is present. Observation of these fields in relation to the double-saddle field antenna shows that the two large peaks occur directly under the azimuthal straps of the antenna, and additionally, since the current in these two straps is opposite in direction, we expect a $\pi$ phase change. The decrease in field magnitude seen for cases B and C compared to case A in Fig. 4 is likely due to the change in skin depth associated with the increased density, as well as the increase in antenna resistance, and hence decrease in antenna current (since the near fields are proportional to the applied antenna current) previously observed. ${ }^{27}$ By measuring the current in the antenna for the no plasma condition (case D), and by making use of a simple application of the Biot-Savart law applied to the azimuthal straps of the antenna, we would expect a maximum magnetic field of the order of $100 \mu \mathrm{T}$, consistent with the measurements made for case D. Although some slight differences are present between each of the cases in Fig. 4, we see that as a whole the near fields of the antenna are sufficiently strong that they are hiding or obscuring any wave fields that may be present. This is especially true if as Sec. IV A suggests, a wave with wavelength of $0.2 \mathrm{~m}$ is being excited for case B, so that maxima and minima in the axial profile of $B_{z}$ would occur in similar locations to those of the antenna near fields.

Measurements of wave fields within the source region proved very difficult, and interference from the near fields of the antenna was consistently observed. Probes needed to be positioned in such a way so as to allow cancellation of the antenna fields (due to opposite fields produce by different parts of the antenna). This proved difficult due to the geometry and setup of the reactor/probes. Radial measurements were eventually obtained, as shown in Fig. 5 , at $z=-16 \mathrm{~cm}$ along a radial line such that the azimuthal straps of the antenna were close to being equidistant from this line (since the current in these straps is in the opposite sense to each other, the $B_{z}$ component of the near fields should cancel along this line). Because of difficulties in positioning the probe accurately, and a slight misalignment of the antenna/source tube, the field cancellation was not complete. Figure 5 shows radial profiles for case A (closed triangles) and case B (closed circles). Profiles for case $\mathrm{C}$ and the no plasma case (case D) are similar to case A and are not shown for clarity. The measurements for case B shows a double-peaked profile characteristic of an $m=1$ helicon mode, with a $\pi$ phase change occurring at the minimum $(r=0 \mathrm{~cm})$. The radial wave fields in simple helicon systems are Bessel functions. The solid curve in Fig. 5 shows a best fit curve of a combination of $J_{0}$ and $J_{1}$ Bessel functions (the $J_{0}$ Bessel function has only a small component $\approx 4 \%$, which could be present due to the nonsymmetric nature of the antenna, or could be a residual 


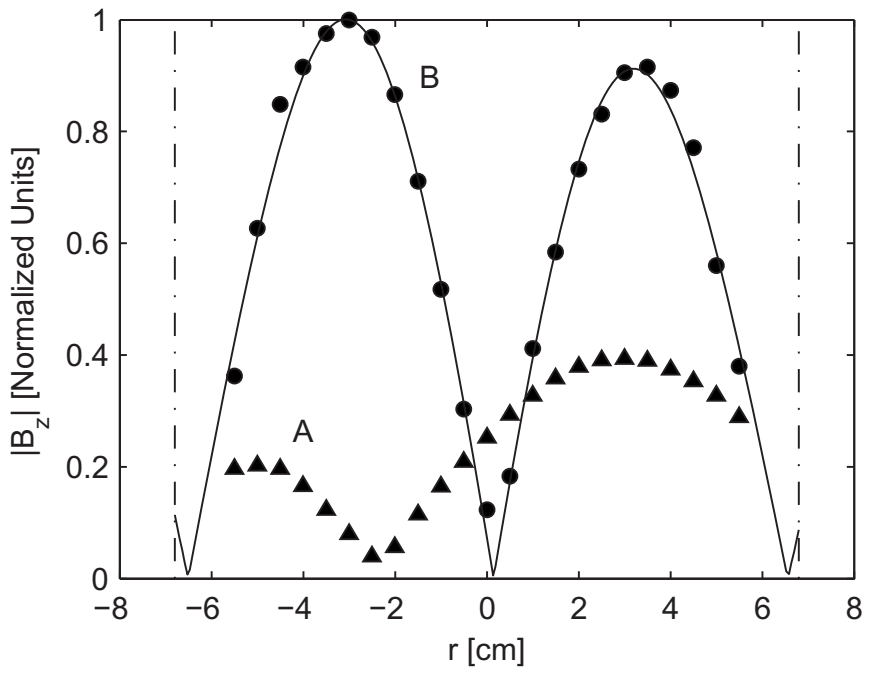

FIG. 5. Radial profile of the amplitude of $B_{z}$ in the source region at $z=-16 \mathrm{~cm}$ for case A (closed diamonds) and case B (closed circles) in Fig. 3(a). Case $C$ and the no plasma case (case D) give similar behavior to case $\mathrm{A}$ and are not shown for clarity. The measurements are taken along a radial line such that the azimuthal straps of the double-saddle field antenna are close to being equidistant from the line. The vertical dashed lines mark the source tube boundaries, while the solid black line is a best fit curve based on Bessel functions.

effect of the incomplete cancellation of the antenna fields). From the fitted curve, we estimate a perpendicular wave number of $k_{\perp}=58.6 \mathrm{~m}^{-1}$.

As discussed above, the difficulty with taking wave measurements in the source region is related to interference by the antenna fields. Thus, to diagnose the waves further, we take B-dot probe measurements far from the antenna where the near fields should have decayed sufficiently. The magnitude of the rf fields in the downstream region is shown in Fig. 6. Here again we see that aside from changes in the magnitude, the fields show essentially the same behavior, with the magnitude decaying rapidly with distance away from the source region. This once again suggests that the observed behavior is primarily linked to the near fields of the antenna and does not provide evidence for any wave fields for case B. At first, this appears strange, especially given the many similarities present with the density peaks observed in uniform magnetic fields (where helicon wave behavior has been measured $^{6,10}$ ), as well as the fact that $m=1$ wave signatures were measured in Fig. 5. However, by observing the axial profile of the applied magnetic field (open diamonds) shown in Fig. 7(a), we note that at $z=-2.5 \mathrm{~cm}$ the field strength is $0.48 \mathrm{mT}$. This yields an electron cyclotron frequency of $13.56 \mathrm{MHz}$, which is equal to the applied rf antenna frequency. Thus, an ECR region exists within the system close to the source tube exit. It is well known from helicon theory ${ }^{2}$ that right hand polarized (RHP) waves cannot propagate past such a resonance point. Thus, it is possible that a wave mode does indeed exist for case $\mathrm{B}$, but that waves are trapped within the source region. If this is the case, then it suggests that by shifting the ECR point further into the diffusion chamber, the propagation of waves downstream could be allowed.

To test this, a number of magnetic field configurations

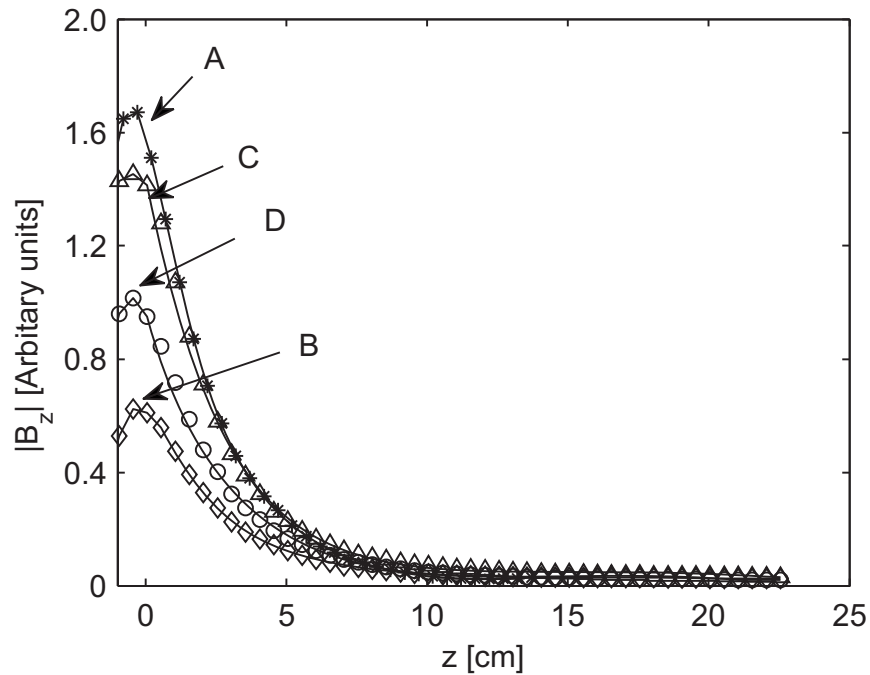

FIG. 6. Axial profile of the amplitude of $B_{z}$ in the diffusion chamber at $r=4.8 \mathrm{~cm}$ for case A (stars), case B (open diamonds), case C (open triangles), and case D (open circles) in Fig. 4.

(designated as cases 1-5) are used, as shown in Fig. 7(a), which aim to leave the magnetic field within the source region the same, while only changing the field strength/ geometry in the downstream region. To accomplish this, use is made of a combination of all four magnetic field coils shown in Fig. 1. The original magnetic field configuration [open diamonds in Fig. 7(a)] applies to point B in Fig. 3(a). This configuration is now designated case 1 , and subsequent configurations [shown in Fig. 7(a)] are designated cases 2-5. The axial density profiles, together with the downstream wave fields for each of the test cases, are shown in Figs. 7(b)-7(d). The vertical arrows serve as a visualization aid and show the behavior of the plasma density and wave fields as the magnetic field is changed. Here it can be seen that as the magnetic field near the source tube exit $(z \approx 0 \mathrm{~cm})$ is increased, wave fields now become apparent in the downstream region [Fig. 7(c)], and this is correlated with an increase in the downstream plasma density and a decrease in the upstream density [Fig. 7(b)]. The plasma density in the source drops from around $2.2 \times 10^{17} \mathrm{~m}^{-3}$ for case 1 (open diamonds) to $1.1 \times 10^{17} \mathrm{~m}^{-3}$ for case 5 (open triangles), while at the same time the downstream density increases by a factor of almost 6 . In particular, the density gradient begins to decrease rapidly, until for case 5 an almost axially uniform density profile is present.

Figure 7(c) shows that as the magnetic field is changed, wave fields start becoming evident, increasing in magnitude and axial extent for each further change to the magnetic field. This behavior, together with the observed density profile changes, suggests that waves are present in the source region, and that the magnetic field near the source exit acts almost like a valve. As the valve is opened, waves that were previously trapped in the source region are now able to travel downstream and can thus deposit more of their energy in this region as opposed to the source region (hence, the decrease in upstream density). As the waves are able to travel further downstream, they will eventually encounter the grounded metal back plate at the end of the diffusion chamber. The 

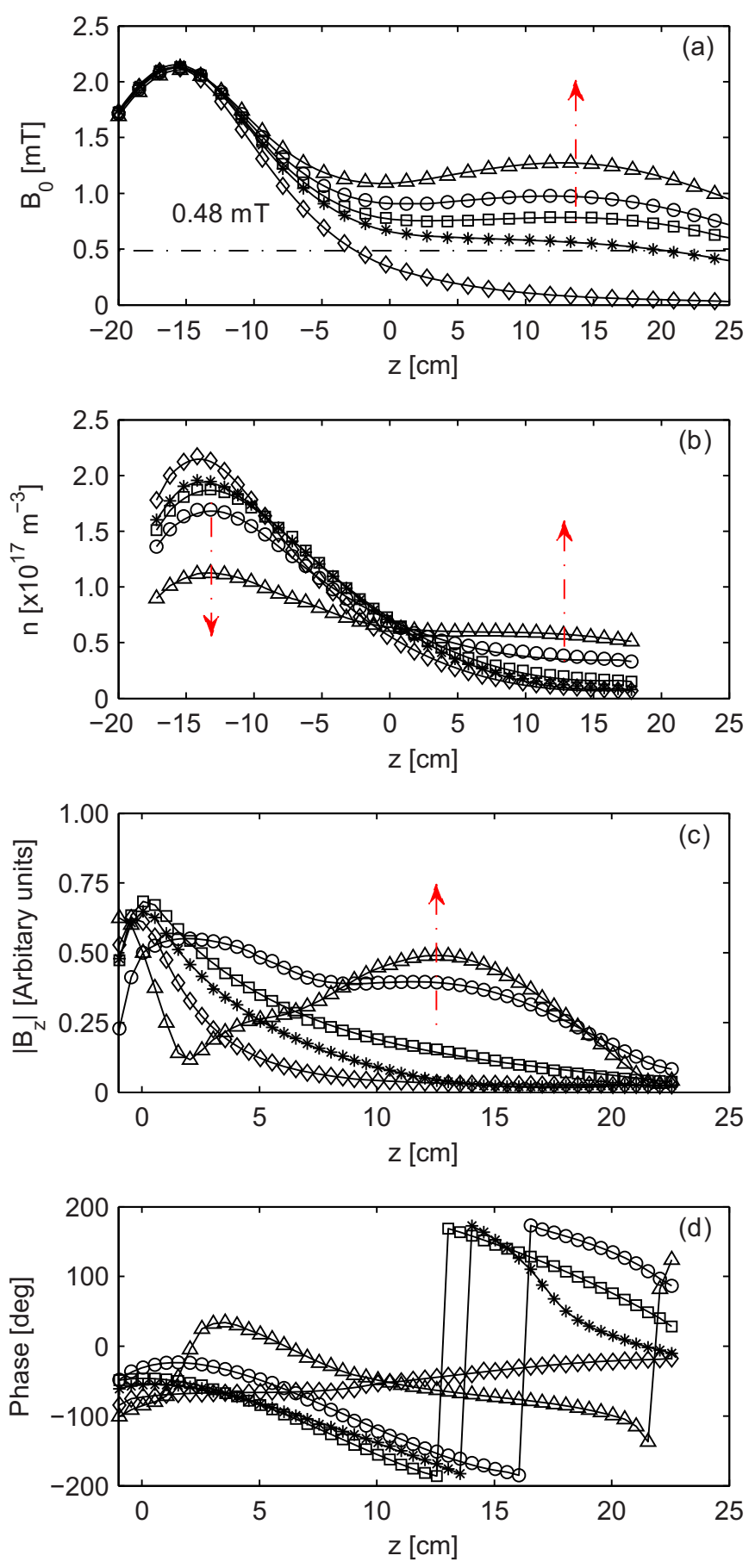

FIG. 7. (Color online) (a) Calculated axial profiles of the applied magnetic field, with case 1 (open diamonds, and point B of Fig. 3), case 2 (stars), case 3 (open squares), case 4 (open circles), and case 5 (open triangles). The horizontal dotted line shows the magnetic field giving an electron cyclotron frequency of $13.56 \mathrm{MHz}$. (b) Axial plasma density profiles for the cases in (a). (c) Axial profiles of the amplitude of $B_{z}$ at $r=4.8 \mathrm{~cm}$ for the cases in (a). (d) Axial profiles of the phase variation of $B_{z}$ for the cases in (a). The vertical arrows in (a)-(c) are for visualization purposes and show the system response as the applied magnetic field is changed according to (a).

definite peak at $z=12.5 \mathrm{~cm}$ observed in the wave fields for case 5 suggests that the waves have reached this back plate, and that some type of wave reflection (leading to interference effects) could be occurring, ${ }^{32}$ although this has presently not yet been investigated further.
For cases 2-4 in Fig. 7(d) traveling wave behavior appears present, and an estimate of the axial wavelength $\lambda_{z}$ can be made from

$$
\lambda_{z}=360\left(\frac{\Delta z}{\Delta \phi}\right)
$$

where $z$ is the axial distance and $\phi$ is the phase variation shown in Fig. 7(d). This gives wavelengths of around 0.32, 0.28 , and $0.31 \mathrm{~m}$ for cases $2-4$, respectively. Since the ratio $B_{0} / n$ (and hence $\omega_{c e} / \omega_{p e}$ ) increases in the downstream region for these cases, from Eq. (1) we would expect a wavelength larger than that within the source region. Thus, these values seem consistent (or at least representative) of an initial upstream wave with a wavelength of approximately $0.2 \mathrm{~m}$.

A valid question to ask at this point is whether or not the plasma stays within the same mode as the magnetic field is changed for the test cases in Fig. 7(a). The reverse power from the directional coupler is monitored for each of the test cases, where the reflected power (which is very low) is seen to remain similar for each case, only beginning to change for case 5. By contrast, the matching changes dramatically during the low magnetic field mode transition of Fig. 3(a). Further increases in the downstream field [after test case 5 in Fig. 7(a)] cause the system to jump out of the mode, and a large change is again seen in the matching. When this happens, the color of the plasma changes substantially, going from a bright pink to a dull purple. As speculated above, the magnetic field near the source exit appears to act like a "valve," and as the valve opens, waves are allowed to escape downstream and are hence able to deposit more of their energy in this region. This, however, results in a decrease of density in the source region, and eventually a point is reached where if the valve is opened further, the density drops to such an extent that the coupling efficiency with the antenna decreases sufficiently that the system is no longer able to sustain a wave mode. From the results of Fig. 7(b), we can estimate this density threshold as around 1 $\times 10^{17} \mathrm{~m}^{-3}$. Thus, in order to delay the onset of this mode loss, we need to keep the density above this value. This can be achieved by operating at higher pressures or by increasing the power input to the system. It is for this reason that measurements here are made at a pressure twice that used in a previous study. ${ }^{27}$ At this original pressure, as the magnetic valve is opened, the system very quickly jumps out of the mode. Only an increase in power at this pressure allows the valve to be opened further. This hypothesis is further strengthened by the fact that as the pressure or power is increased, the maximum value of the density peak is observed to shift to larger magnetic fields [showing an almost linear relationship, consistent with Eq. (1), for a fixed wavelength].

In order to measure the mode structure of the waves formed in the system, radial B-dot probe measurements in the downstream region are carried out at an axial distance of $z=15 \mathrm{~cm}$. Figure 8 shows the $\left|B_{r}\right|,\left|B_{\theta}\right|$, and $\left|B_{z}\right|$ radial profiles for each of the test cases of Fig. 7(a). The inset figures show a schematic of the theoretically expected profiles for an 

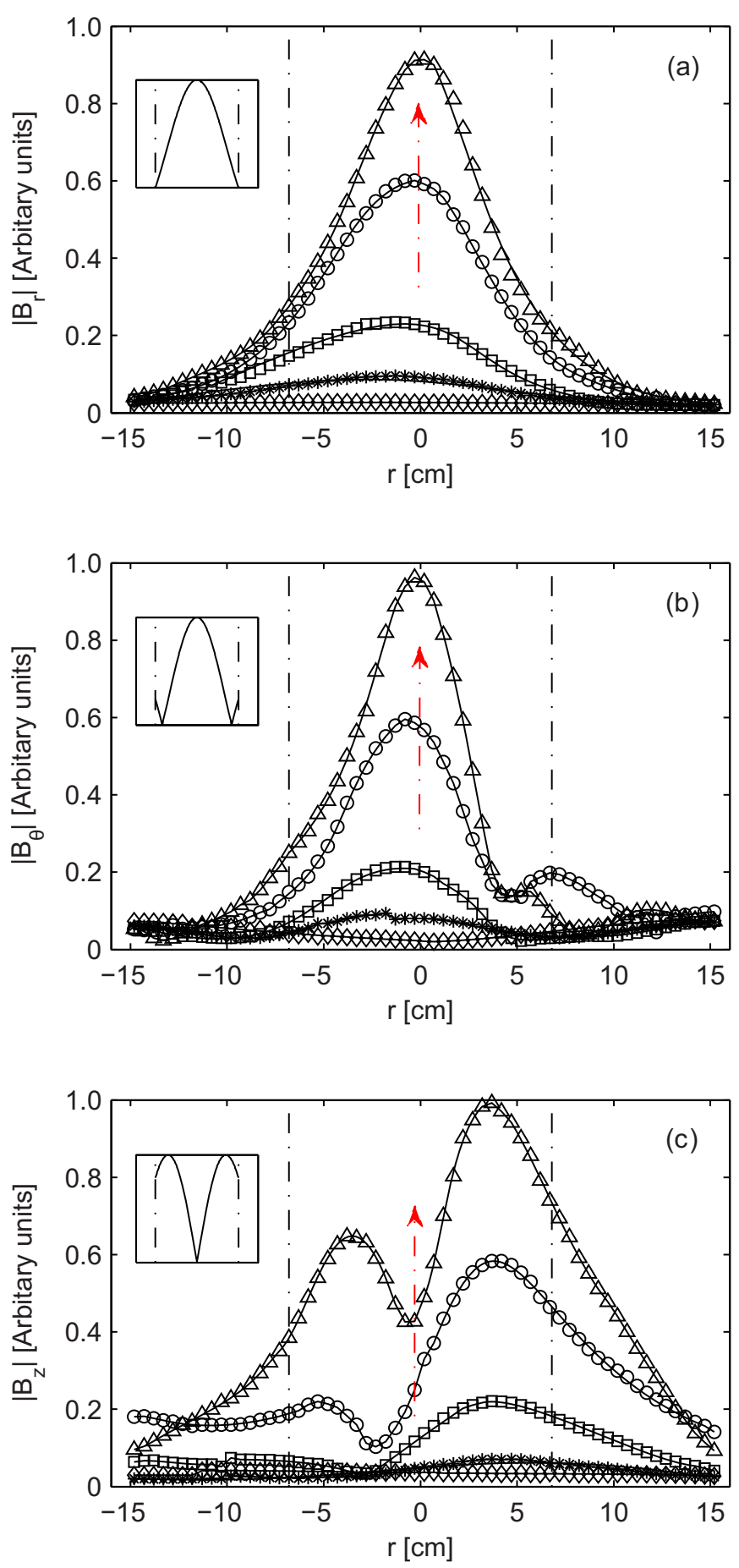

FIG. 8. (Color online) Radial variation at $z=15 \mathrm{~cm}$ of the amplitude of (a) $B_{r}$, (b) $B_{\theta}$, and (c) $B_{z}$ for the magnetic field cases in Fig. 7(a). The inset figures show a schematic of the theoretically expected profiles for an $m=1$ helicon mode. The vertical dotted lines mark the source tube boundary, while the vertical arrows show the wave field response as the applied magnetic field is changed according to Fig. 7(a).

$m=1$ helicon wave. For all three field components, case 1 (open diamonds, and point B in Fig. 3) shows a flat profile with no evidence of any wave fields, but as the magnetic valve is opened, once again wave fields start becoming apparent. The $\left|B_{r}\right|$ profiles show a single large peak centered approximately at $z=0 \mathrm{~cm}$, while the $\left|B_{\theta}\right|$ profiles show a slightly asymmetrical profile, which again contains a central

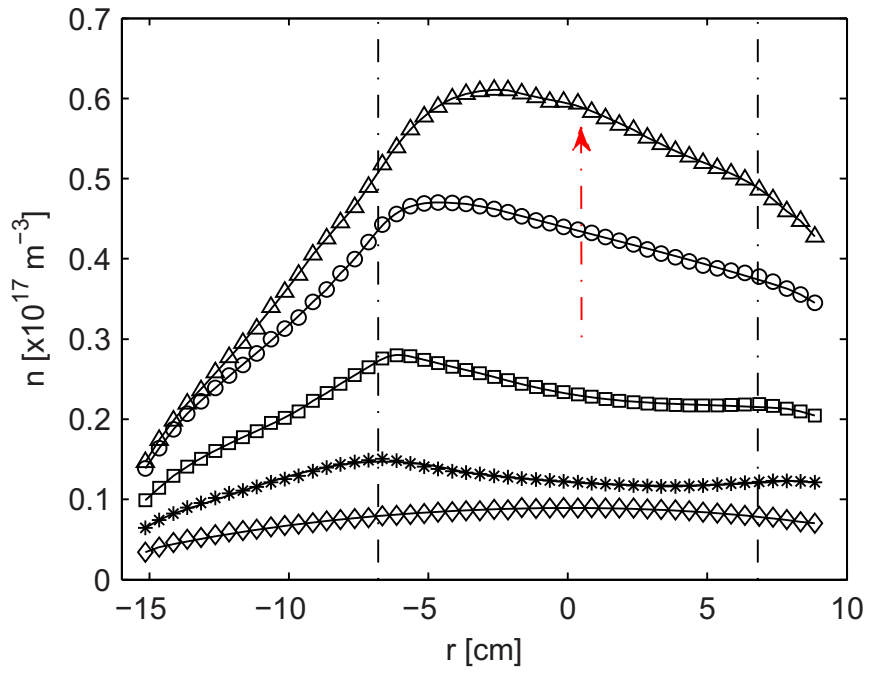

FIG. 9. (Color online) Radial plasma density profiles at $z=15 \mathrm{~cm}$ for the cases in Fig. 7. The arrows show the plasma response as the applied magnetic field is changed according to Fig. 7(a).

peak, with a second smaller peak near $r=5 \mathrm{~cm}$ present for cases 4 (open circles) and 5 (open triangles). These two cases also show a double-peaked $\left|B_{z}\right|$ profile, although again the profile is asymmetrical, with the right hand peak being larger than the left hand peak. It is also noted that the minimum point between these two peaks does not go to zero. A similar result was obtained in Refs. 32 and 33, where it was suggested that an $m=0$ mode could be being excited concurrently with an $m=1$ mode, although the reasons for this still remain unclear. In the present case, a slight eccentricity in the diffusion coils has been observed that could be contributing to this. At any rate, comparison of all three wave components with the theoretically expected profiles show that an $m=1$ mode is most likely dominant. This conclusion is consistent with the $m=1$ wave profile for $B_{z}$ observed in Fig. 5 .

Figure 9 shows the radial density profiles at $z=15 \mathrm{~cm}$, where similar to the wave fields, we see a definite asymmetry. Case 1 (open diamonds) is fairly symmetrical, but as the magnetic valve is opened, an asymmetry in the profiles develops. This suggests another possible reason for the asymmetrical wave field profiles in Fig. 8 since the density profiles would affect the resulting wave field shape. The increase in density in the downstream region is most clearly seen in Fig. 9, where the density for case 5 (open triangles) is almost six times larger than that for case 1 .

In order to confirm that it is, in fact, the ECR region near the source exit that is important for the valvelike behavior and not the magnetic field in the downstream region, we ran several further magnetic field test cases, as shown in Fig. 10(a). Here the magnetic field in the source region (including the source exit) is kept essentially the same, but only the downstream field is changed. Figure 10(b) shows the axial density profiles, while Fig. 10(c) shows the axial wave field profiles. Here we see that the density profiles remain virtually identical, and that no significant wave fields are present. Again no change in the system matching is seen. This suggests that to a large extent, as long as the ECR region near the source exit remains fixed, changes in the downstream 

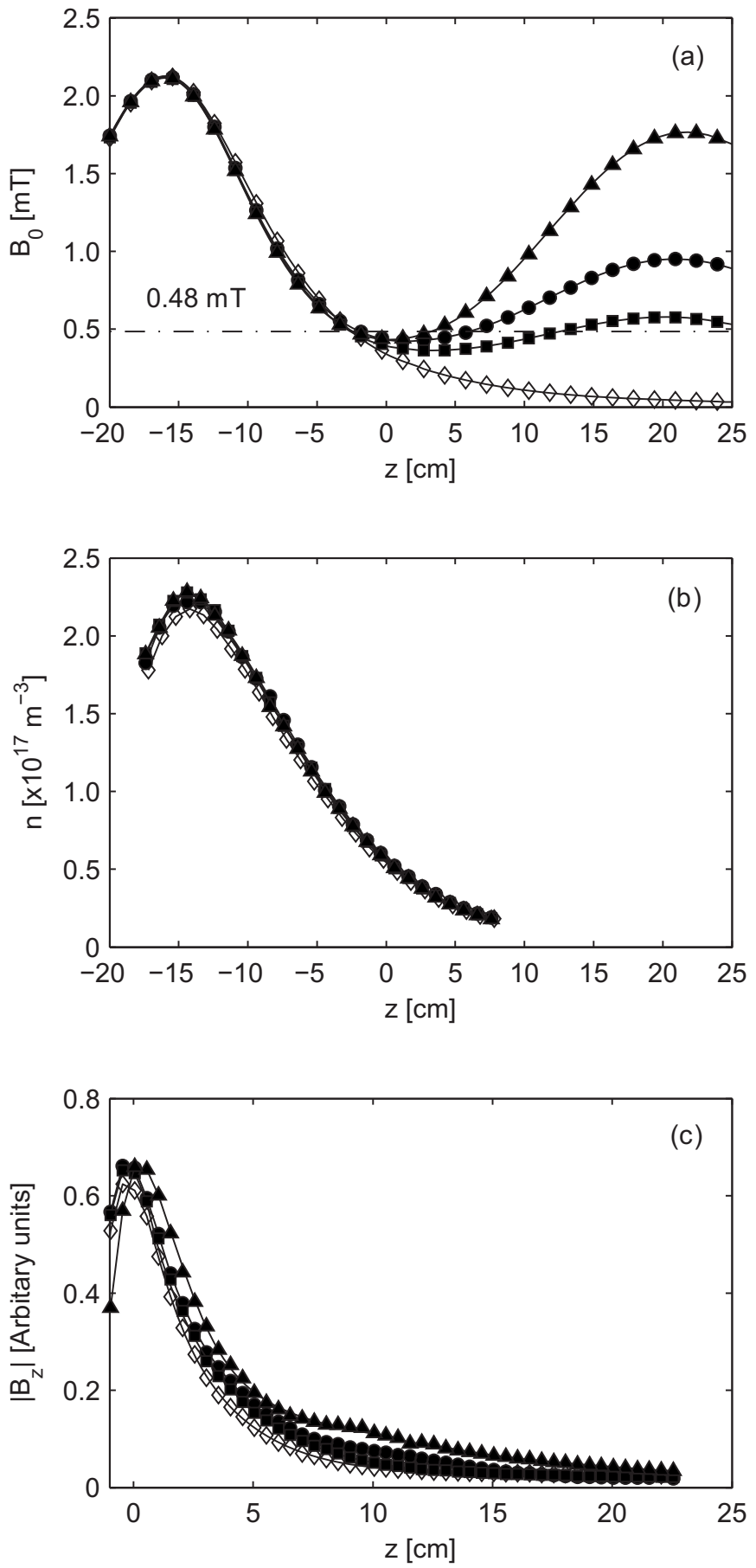

FIG. 10. (a) Additional applied magnetic field test cases with case 1 (open diamonds), case 6 (closed squares), case 7 (closed circles), and case 8 (closed triangles). (b) Axial density profiles for the cases in (a). (c) Axial profiles of the magnitude of $B_{z}$ at $r=4.8 \mathrm{~cm}$ for the cases in (a).

field cannot affect the upstream plasma production or wave propagation. Thus, it is as if the plasma is in a "choked" condition.

\section{Plasma potential and ion beam behavior}

Using RFEA (A) (see Fig. 1) the axial plasma potential profiles are established for each of the cases in Fig. 7(a). By taking the derivative of the ion current as a function of the discriminator voltage (swept voltage applied to one of the

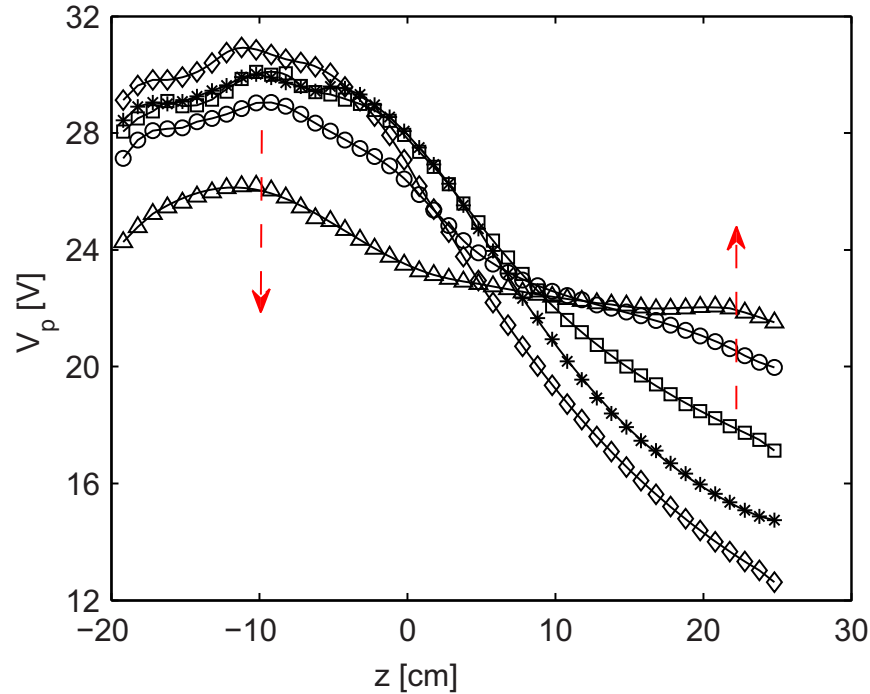

FIG. 11. (Color online) Axial plasma potential profiles for the cases in Fig. 7(a), measured with RFEA (A) in Fig. 1. The vertical arrows show the plasma response as the applied magnetic field is changed according to Fig. 7(a)

RFEA grids), the ion energy distribution function (IEDF) can be established at each axial location. ${ }^{24}$ Since the orifice of RFEA (A) faces the walls of the reactor, it can only see a single ion population centered on the local plasma potential. A Gaussian function is fitted to this IEDF, and the plasma potential is defined by the central value of this Gaussian. ${ }^{24}$ Plasma potential profiles are shown in Fig. 11, with the arrows again showing the behavior as the magnetic field of Fig. 7(a) is changed. In a manner similar to the axial plasma density in Fig. 7(b), as the magnetic field is changed, the plasma potential in the source region decreases, while increasing in the downstream region. From the CP, an upstream electron temperature $T_{e}$ of around $5-6 \mathrm{eV}$ is measured for cases 1-5 in Fig. 7. From theory, for a plasma in contact with a grounded wall (such as the grounded metal grid at one end of the source tube), we would expect a plasma potential of approximately $5.2 T_{e},{ }^{20}$ which for the measured electron temperatures gives values of around $28-31 \mathrm{~V}$, in excellent agreement with the peak upstream potentials in Fig. 11. The drop in plasma potential between locations $z=-10 \mathrm{~cm}$ and $z=20 \mathrm{~cm}$ goes from around $17 \mathrm{~V}$ for case 1 (open diamonds) to around $2 \mathrm{~V}$ for case 5 (open triangles), thus giving an essentially flat axial profile.

We have shown previously ${ }^{27}$ that associated with the low magnetic field mode of Fig. 3(a) is the existence of an ion beam in the downstream region. It is thus of interest to see how this ion beam is affected as the magnetic field is changed according to Fig. 7(a). To investigate this, we make use of the dogleg RFEA, which has its orifice facing the source tube exit. The total collected ion current as a function of discriminator voltage is plotted in Fig. 12(a), together with its derivative (the IEDF) in Fig. 12(b), taken at $z=15 \mathrm{~cm}$. Case 5 (open triangles) shows only a single ion population, while case 1 (open diamonds) shows a much broader distribution with a fraction of higher energy ions. As the magnetic 

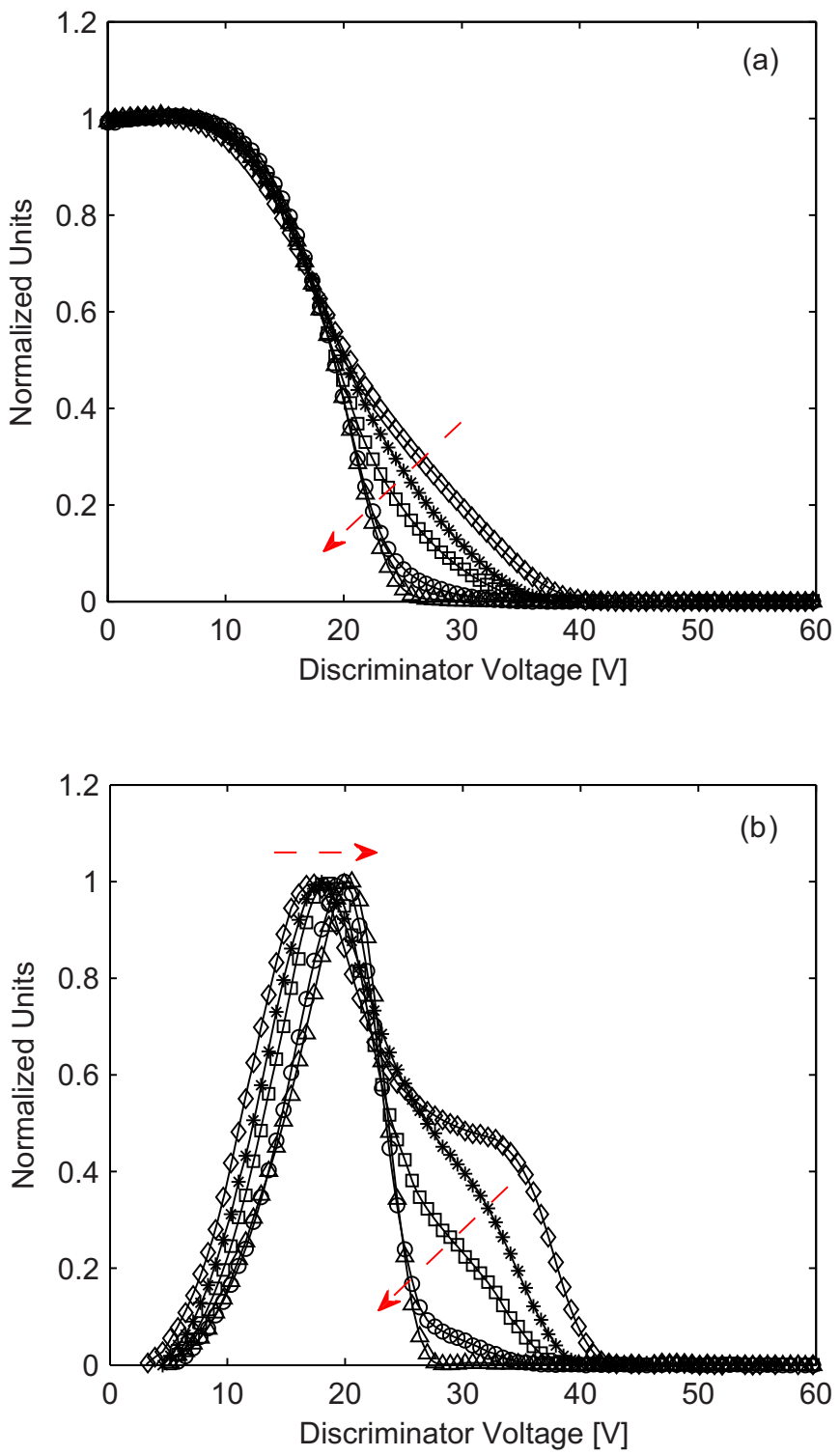

FIG. 12. (Color online) Normalized ion current (a) and IEDF (b) at $z=15 \mathrm{~cm}$ and $r=0 \mathrm{~cm}$ for the cases in Fig. 7(a), measured with the dogleg RFEA in Fig. 1. The arrows show the plasma response as the applied magnetic field is changed according to Fig. 7(a).

field is changed, this group of higher energy ions begins to disappear, until for case 5 they are gone altogether. It is also seen that the central value of the main ion population shifts to higher potentials (from around 16 to $21 \mathrm{~V}$ ), in agreement with the plasma potential results in Fig. 11. From Fig. 10, the IEDFs for all three additional magnetic field test cases remain virtually identical to that of case 1 . It has been shown previously that an important factor in ion beam formation in these low pressure plasmas is the existence of a sufficiently large density gradient. ${ }^{27,34}$ From Fig. 7, as the magnetic field changes, the density gradient between the source and the downstream regions begins to decrease, correlating with the decreased plasma potential drop, and hence the lack of a sufficient accelerating mechanism for the ions. ${ }^{35}$

\section{DISCUSSION}

Although the results presented above seem to suggest that waves can be made to travel downstream by changing the magnetic field at the source exit, a number of factors remain unknown. It is unclear whether waves are being reflected at the source exit or whether they are being rapidly attenuated by the ECR region present there. Since the opposite end of the source tube is terminated with a metal grid, waves would undergo reflection here, so that they could become trapped in the source region if they were reflected near the source tube exit as well. Additionally, since the source tube length is approximately $0.2 \mathrm{~m}$, which is close to the estimated wavelength of $0.2 \mathrm{~m}$ from Sec. IV A, a wavelength would fit inside the source region, and so the system could be exhibiting resonatorlike behavior.

Budden $^{36}$ obtained an analytical solution for RHP waves incident on an isolated infinity (such as at a cyclotron resonance point) in the refractive index and found that no wave reflection occurs, only absorption takes place. However, the solution assumes a slowly varying cold plasma, and, in particular, a linear magnetic field. In the present case, however, the applied magnetic field used and the resulting density profiles contain sharp gradients close to the source exit, and it is unclear how the analytical results would change under these circumstances. In addition, the cold plasma assumption used ignores the axial electron velocity, and hence precludes any Doppler shifting effects from occurring. Because electrons have a velocity component $v_{z}$ parallel to the direction of wave propagation, within the electron's frame of reference a different wave frequency $\bar{\omega}$ is observed, which can be given by

$$
\bar{\omega}=\omega-k_{z} v_{z} .
$$

The consequence of this Doppler-shift is that electrons with different velocities require a different magnetic field in order to undergo cyclotron resonance (i.e., resonance now occurs at $\left.\omega_{c e}=\bar{\omega}\right)$. At wave frequencies close to the electron cyclotron frequency, it is well known that RHP waves can readily become attenuated due to spatial Doppler-shifted cyclotron damping. ${ }^{37,38}$ In order to gain some insight into the possible importance of these Doppler shifting effects, we consider the warm plasma dispersion relation ${ }^{39}$

$$
1-\frac{k_{z}^{2} c^{2}}{\omega^{2}}+\frac{\omega_{p e}^{2}}{\omega k_{z} v_{t}} Z(\xi)=0
$$

where the wave number is now complex, $k_{z}=k_{r}+i k_{i}$, $v_{t}=\sqrt{2 q T_{e} / m}$ is the mean electron speed, $q$ and $m$ are the electron charge and mass, respectively, $\xi=\left(\omega-\omega_{c e}+i \nu\right) / k_{z} v_{t}$, $\nu$ is the electron collision frequency, and $Z(\xi)$ is the plasma dispersion function. In Eq. (5) the ions are assumed to be immobile, the electrons have a Maxwellian distribution, and the plasma density and external magnetic field are uniform (a more complete analysis of spatial cyclotron damping has been carried out by Olson, ${ }^{40}$ including effects associated with the excitation of waves, but this is beyond the scope of the present paper). Using a representative plasma density of $1.5 \times 10^{17} \mathrm{~m}^{-3}$ (for case 1 in Fig. 7), and an electron temperature of $6 \mathrm{eV}$, we can solve Eq. (5) for a wave frequency 

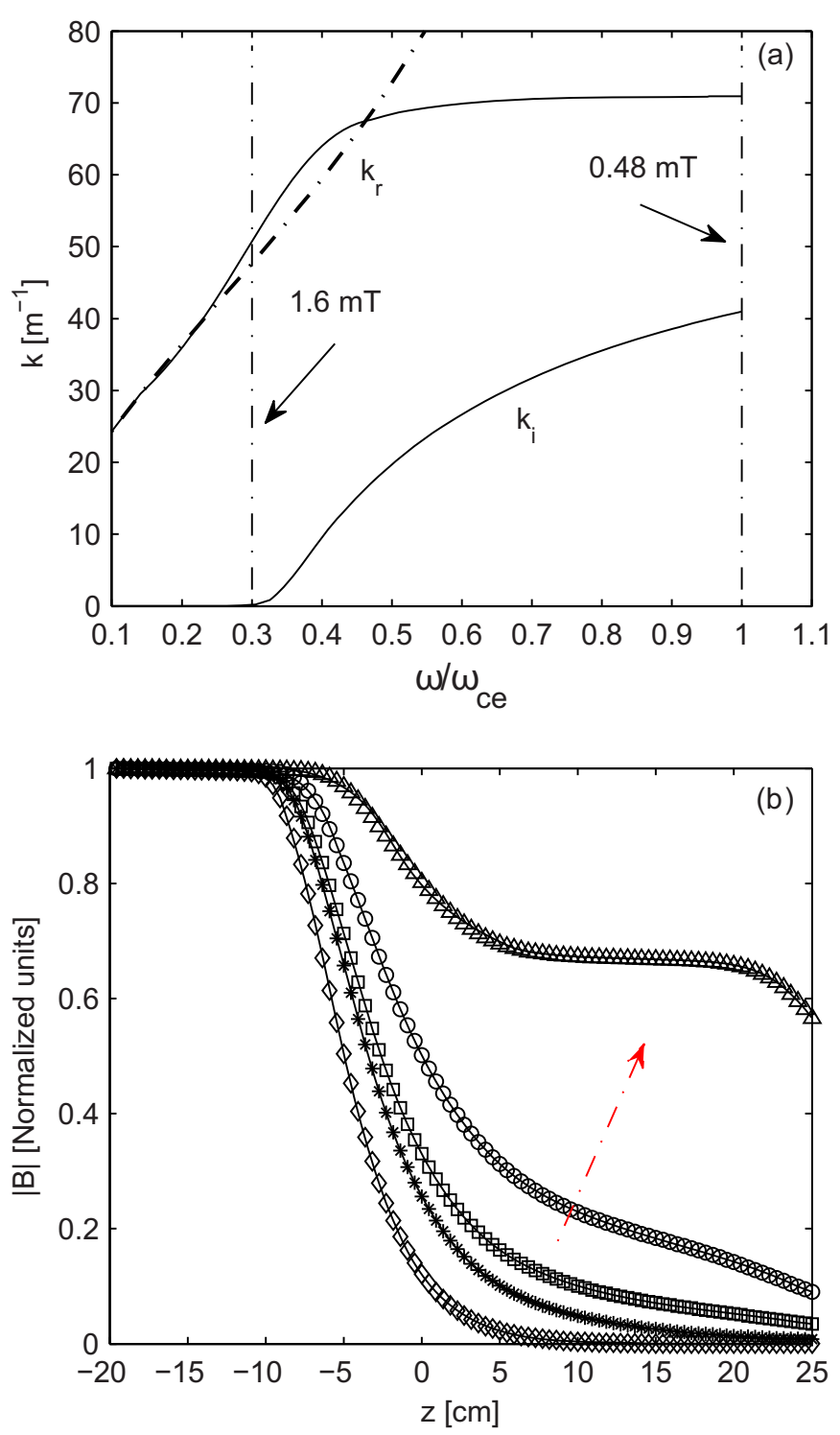

FIG. 13. (Color online) (a) Real $\left(k_{r}\right)$ and imaginary $\left(k_{i}\right)$ parts of the wave number from Eq. (5) for a frequency of $13.56 \mathrm{MHz}$ as a function of magnetic field $\left(\omega / \omega_{c e}\right)$. The vertical dashed lines show the magnetic field values between which damping occurs within the figure, while the bold dashed curve shows the cold plasma dispersion relation, obtained by letting $T_{e} \rightarrow 0$ in Eq. (5). (b) Spatial wave damping for the magnetic field test cases in Fig. 7(a) using magnetic field and plasma density profiles from Figs. 7(a) and 7(b), and wave damping values obtained from Eq. (5). The markers on the curves correspond to the cases in Fig. 7(a), that is, case 1 (open diamonds), case 2 (stars), case 3 (open squares), case 4 (open circles), and case 5 (open triangles). The arrow shows the wave amplitude response as the magnetic field is changed according to Fig. 7(a).

of $13.56 \mathrm{MHz}$ as a function of magnetic field $\left(\omega / \omega_{c e}\right)$. This is shown for both the real and imaginary parts of the wave number in Fig. 13(a) [the bold dashed curve shows the cold plasma dispersion relation obtained by letting $T_{e} \rightarrow 0$ in Eq. (5)].

The appearance of an imaginary part to the wave number in Fig. 13(a) is associated with the collisionless damping due to cyclotron resonance. Of particular interest in this regard is the fact that a true resonance no longer exists (i.e., $k_{r}$ remains finite), as well as the fact that cyclotron damping begins for $\omega / \omega_{c e} \approx 0.3$. This is in contrast to much higher wave frequencies $(>1 \mathrm{GHz})$, where damping begins for $\omega / \omega_{c e} \approx 0.8-0.9 .^{37,38}$ The vertical dashed lines in Fig. 13(a) show the range of magnetic field values between which damping is present in the figure. Observation of case 1 (open diamonds) in Fig. 7(a) shows that this range of magnetic field values is present within a fairly large part of the source region. If we use an average value of the wave damping (say $k_{i} \approx 30 \mathrm{~m}^{-1}$ ), then we obtain an e-folding of $\approx 3 \mathrm{~cm}$ (since damping adds an exponential decay factor to the wave amplitude). This suggests that the waves would be strongly damped by the time they reach the diffusion chamber and is consistent with the lack of waves observed for case 1 in Fig. 7(c). Equation (5), however, assumes a uniform magnetic field and plasma density, and we have not yet solved the Vlasov equation to account for these inhomogenieties; however, we can make a crude estimate as follows. Using the magnetic field and density profiles from Figs. 7(a) and 7(b) (and extrapolating the density for $z>18 \mathrm{~cm}$ ), we can solve Eq. (5) and establish a value for the wave damping at each axial location. To do this, we use a discrete axial coordinate $z_{n}$ with uniform spacing between each point (i.e., $\left.\Delta z=z_{n}-z_{n-1}\right)$. By then assuming an initial upstream wave $|B|$, with amplitude of 1 (and for the sake of argument ignoring the change of amplitude due to the change of plasma dielectric), we can find the amplitude at each axial location $z_{N}$ from $\left|B\left(z_{N}\right)\right|=\prod_{n=1}^{N} \exp \left(-k_{i}\left(z_{n}\right) \Delta z\right)$, where $k_{i}\left(z_{n}\right)$ is the wave damping at $z_{n}$ [obtained from Eq. (5)]. Doing this for each of the test cases in Fig. 7, we produce Fig. 13(b). Comparison with Fig. 7(c) shows there to be fairly reasonable qualitative agreement in the downstream region. As the magnetic field is changed according to Fig. 7(a), waves become less damped and are able to travel deeper into the downstream region. Case 1 is sufficiently strongly damped well before entering the diffusion chamber, while the damping decreases significantly for case 5 , so that waves are able to penetrate deep into the diffusion chamber and reach the back plate (where wave reflection could occur, as was discussed above in Sec. IV B). We are, however, cautious to draw definite conclusions from this crude calculation, since of course the nonuniformities in magnetic field and plasma density have not been correctly accounted for. Nevertheless, the above argument does suggest that Doppler-shifted cyclotron damping could be a feasible mechanism involved in the present investigation.

Simulation of helicon wave propagation in a plasma with sharp density and magnetic field gradients was performed by Mouzouris and Scharer, ${ }^{16}$ where it was found that the axial inhomogenieties caused distributed reflections of any incident helicon waves, thus preventing helicon propagation. However, these simulations were done at larger magnetic fields $\left(B_{0}>20 \mathrm{mT}\right)$ so that no cyclotron resonance would be expected in the system. Experimental work by Guo et al. ${ }^{17}$ on helicon propagation in nonuniform magnetic fields included some cases with an ECR region present, yet no ECR heating was observed. It was suggested that the strong magnetic field gradients present could change the phase correlation between the electrons and helicon wave sufficiently over a gyroperiod so that no significant cyclotron heating would occur. Additionally, the wave fields produce a changing axial 


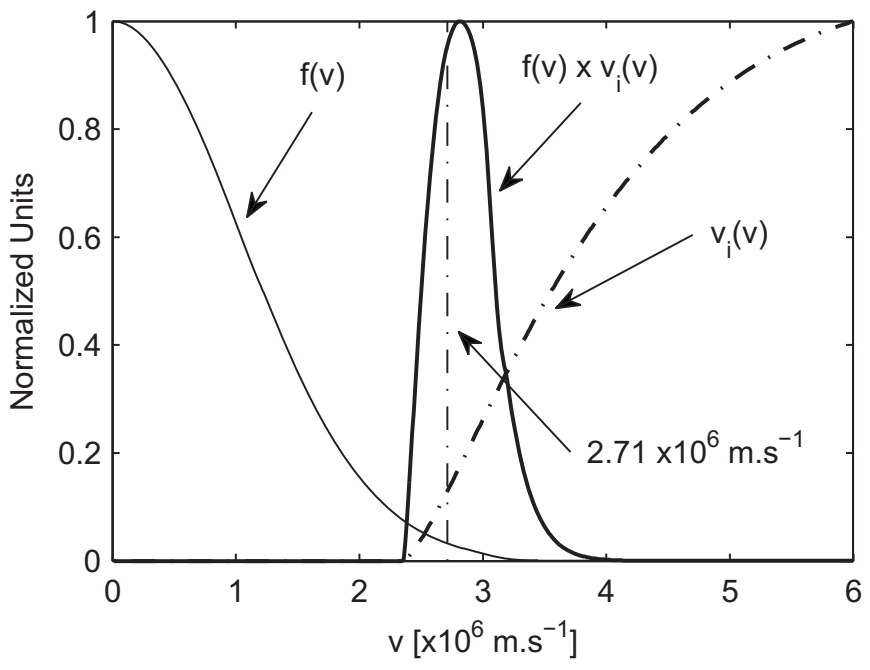

FIG. 14. Normalized curves showing a $6 \mathrm{eV}$ electron distribution function (light solid line), $f(v)$, velocity dependent ionization rate for argon (bold dashed curve), $\nu_{i}(v)$, and the product of these two curves showing which electrons in the distribution are most effective in causing an ionizing event (bold solid line). The vertical dotted line shows the phase velocity of a helicon wave with a frequency of $13.56 \mathrm{MHz}$ and a wavelength of $0.2 \mathrm{~m}$.

magnetic field component, and these were found to be of a similar magnitude to that of the applied magnetic field in the ECR region so that the cyclotron resonance process would likely be strongly hindered. From Fig. 4(a), the magnitude of the wave fields near the ECR region are much smaller than the applied magnetic field $[<10 \%$, see also Fig. $7(\mathrm{a})]$, so we would not expect the same disruption in the present system.

Degeling et al. ${ }^{10}$ noted a distinct correlation between the phase velocity of helicon waves and the velocity of electrons of a Maxwellian distribution most effective in causing ionization. Using a double half-turn antenna, $m=1$ helicon waves with wavelengths between 10 and $60 \mathrm{~cm}$ were excited in a large plasma diffusion system (of a larger scale than the present reactor). For helicon waves with phase velocities of around $3 \times 10^{6} \mathrm{~m} \mathrm{~s}^{-1}$, a large increase in the downstream plasma density was observed. It was suggested that the parallel component of the helicon waves $E_{z}$ was causing particle trapping, and, in particular, that the wave was interacting with electrons with velocities close to the wave velocity (this is in contrast to the mechanism of Doppler-shifted cyclotron damping, which is an interaction between electrons and the perpendicular component of the wave field).

By performing a similar analysis to Degeling et al. ${ }^{10}$ we obtain Fig. 14. Here we have taken a $6 \mathrm{eV}$ electron distribution function (representative of the measurements made in the present system), $f(v)$, and multiplied it by the ionization collision frequency for argon (using standard cross sections and a pressure of $0.08 \mathrm{~Pa}), \nu_{i}(v)$, to obtain the bold solid curve in Fig. 14. The vertical dashed line shows the phase velocity of a helicon wave with wavelength of $0.2 \mathrm{~m}$. Here, similar to Degeling et al., ${ }^{10}$ we note a strong correlation between the wave phase velocity and the velocity of electrons most effective at causing an ionizing event. This suggests that wave-particle trapping due to the axial component of the electric field of the helicon wave could be relevant to the present system.
Degeling $^{41}$ also performed numerical modeling of particle trapping by helicon waves. Perturbations in the electron distribution function due to a parallel electric field $E_{z}$ were built up by considering trajectories of individual electrons in an imposed wave field. The results showed that the ionization rate is highest when the phase velocity is close to $(2-3) \times 10^{6} \mathrm{~m} \mathrm{~s}^{-1}$, where electrons can interact strongly with the wave. Particle trapping was also observed to occur in standing helicon waves. Since a standing wave is a combination of forward and reverse waves, electrons from both the positive and negative sides of the distribution function can now be involved in trapping mechanisms with these waves, respectively. We might then wonder whether such a mechanism could occur in the present system if wave reflection was to happen at the source tube exit. The trapping length $L_{\mathrm{tr}}$ is a measure of the distance needed for significant perturbations in the distribution function to develop due to wave trapping and can be given by ${ }^{41}$

$$
L_{\mathrm{tr}}=v_{\phi} \tau_{\mathrm{tr}}=2 \pi v_{\phi}\left(\frac{m}{q k_{z} E_{0}}\right)^{1 / 2},
$$

where $\tau_{\mathrm{tr}}$ is the trapping period and $E_{0}$ is the amplitude of the axial component of the helicon electric field. Using a wave amplitude of the order of $E_{0} \approx 100 \mathrm{~V} \mathrm{~m}^{-1}$, we estimate a trapping length of $L_{\mathrm{tr}} \approx 0.7 \mathrm{~m}$. This is the distance needed for a trapped electron to undergo a complete oscillation; however, only $L_{\mathrm{tr}} / 4=0.18 \mathrm{~m}$ (which is of the order of the source tube length) is needed for a trapped electron to be accelerated to the wave phase velocity. Within distances of this order, Degeling ${ }^{41}$ showed that a large amount of power absorption can take place well before complete trapping has set in.

\section{CONCLUSIONS}

In summary, we have shown that helicon wave propagation can be controlled in a low nonuniform (diverging) magnetic field. The presence of an ECR region near the source exit prevents the propagation of waves into the downstream region, but by modifying the applied field, waves can be made to escape. Radial measurements taken with a B-dot probe suggest that the observed waves are most likely an $m=1$ mode. Associated with the observation of these wave fields in the downstream region are corresponding changes in both the plasma potential and density, suggesting that the wave power deposition profile can, to a certain extent, be controlled. As waves are able to escape into the downstream region, the density and plasma potential profiles becomes flatter, and the presence of an ion beam in the downstream region disappears. We have presented evidence to support both Doppler-shifted cyclotron damping and wave-particle trapping interactions as important mechanisms in the present study, and future work will aim to investigate these phenomena further.

\section{ACKNOWLEDGMENTS}

The authors would like to thank M. Lieberman for reading the manuscript and offering some useful suggestions. 
${ }^{1}$ R. W. Boswell, Plasma Phys. Controlled Fusion 26, 1147 (1984).

${ }^{2}$ R. W. Boswell and F. F. Chen, IEEE Trans. Plasma Sci. 25, 1229 (1997).

${ }^{3}$ F. F. Chen and R. W. Boswell, IEEE Trans. Plasma Sci. 25, 1245 (1997).

${ }^{4}$ F. F. Chen, Phys. Plasmas 10, 2586 (2003).

${ }^{5}$ C. Carter and J. Khachan, Plasma Sources Sci. Technol. 8, 432 (1999).

${ }^{6}$ G. Sato, W. Oohara, and R. Hatakeyama, Plasma Sources Sci. Technol. 9, 3171 (2002).

${ }^{7}$ F. F. Chen, J. Vac. Sci. Technol. A 10, 1389 (1992).

${ }^{8}$ S. Shinohara and T. Soejima, Plasma Phys. Controlled Fusion 40, 2081 (1998).

${ }^{9}$ S. J. Wang, J. G. Kwak, C. B. Kim, and S. K. Kim, Phys. Lett. A 313, 278 (2003).

${ }^{10}$ A. W. Degeling, C. O. Jung, R. W. Boswell, and A. R. Ellingboe, Phys. Plasmas 3, 2788 (1996).

${ }^{11}$ T. Lho, N. Hershkowitz, J. Miller, W. Steer, and G. H. Kim, Phys. Plasmas 5, 3135 (1998).

${ }^{12}$ S. Cho, Phys. Plasmas 13, 033504 (2006).

${ }^{13}$ F. F. Chen, X. Jiang, and J. D. Evans, J. Vac. Sci. Technol. A 18, 2108 (2000).

${ }^{14}$ F. F. Chen and H. Torreblanca, Phys. Plasmas 16, 057102 (2009).

${ }^{15}$ F. F. Chen, IEEE Trans. Plasma Sci. 36, 2095 (2008).

${ }^{16}$ Y. Mouzouris and J. E. Scharer, Phys. Plasmas 5, 4253 (1998).

${ }^{17}$ X. M. Guo, J. Scharer, Y. Mouzouris, and L. Louis, Phys. Plasmas 6, 3400 (1999).

${ }^{18}$ V. F. Virko, K. P. Shamrai, Y. V. Virko, and G. S. Kirichenko, Phys. Plasmas 11, 3888 (2004).

${ }^{19}$ K. Takahashi, C. Charles, R. W. Boswell, T. Kaneko, and R. Hatakeyama, Phys. Plasmas 14, 114503 (2007).

${ }^{20}$ M. A. Lieberman and A. J. Lichtenberg, Principles of Plasma Discharges and Materials Processing (Wiley, New Jersey, 2005).

${ }^{21}$ C. Charles, A. W. Degeling, T. E. Sheridan, J. H. Harris, M. A. Lieberman, and R. W. Boswell, Phys. Plasmas 7, 5232 (2000).
${ }^{22}$ T. Lafleur, C. Charles, and R. W. Boswell, Phys. Plasmas 16, 044510 (2009).

${ }^{23}$ G. D. Conway, A. J. Perry, and R. W. Boswell, Plasma Sources Sci. Technol. 7, 337 (1998).

${ }^{24}$ C. Charles and R. W. Boswell, Phys. Plasmas 11, 1706 (2004).

${ }^{25}$ G. G. Borg and R. C. Cross, Plasma Phys. Controlled Fusion 29, 681 (1987).

${ }^{26}$ C. M. Franck, O. Grulke, and T. Klinger, Rev. Sci. Instrum. 73, 3768 (2002).

${ }^{27}$ T. Lafleur, C. Charles, and R. W. Boswell, Phys. Plasmas 17, 043505 (2010).

${ }^{28}$ A. R. Ellingboe and R. W. Boswell, Phys. Plasmas 3, 2797 (1996).

${ }^{29}$ A. Perry, G. Conway, R. W. Boswell, and H. Persing, Phys. Plasmas 9, 3171 (2002).

${ }^{30}$ F. F. Chen, Plasma Phys. Controlled Fusion 33, 339 (1991).

${ }^{31}$ J. P. Klozenberg, B. McNamara, and P. C. Thonemann, J. Fluid Mech. 21, 545 (1965).

${ }^{32}$ K. K. Chi, T. E. Sheridan, and R. W. Boswell, Plasma Sources Sci. Technol. 8, 421 (1999).

${ }^{33}$ G. Sato, W. Oohara, and R. Hatakeyama, J. Phys. Soc. Jpn. 75, 043501 (2006).

${ }^{34}$ C. Charles and R. W. Boswell, Appl. Phys. Lett. 91, 201505 (2007).

${ }^{35}$ C. Charles, J. Vac. Sci. Technol. A 11, 157 (1993).

${ }^{36} \mathrm{~K}$. G. Budden, Radio Waves in the Ionosphere (Cambridge University Press, London, 1961).

${ }^{37}$ C. Christopoulos, R. W. Boswell, and P. J. Christiansen, Phys. Lett. 47A, 239 (1974).

${ }^{38}$ B. McVey and J. Scharer, Phys. Rev. Lett. 31, 14 (1973).

${ }^{39}$ D. G. Swanson, Plasma Waves (IOP, London, 2003).

${ }^{40}$ C. L. Olson, Phys. Fluids 15, 160 (1972).

${ }^{41}$ A. W. Degeling, Ph.D. thesis, Australian National University, 1999. 